\title{
IMPROVE GAMUT MAPPING OF COLOR MANAGEMENT SYSTEM BY PERCEPTUAL QUALITY-ORIENTED ANALYSIS
}

\author{
Shaohua CHEN, and Azeddine BEGHDADI
}

Lab L2TI, University Paris13, France

\begin{abstract}
The state-of-the-art solution for Color Management System (CMS) proposed by ICC V4 is mainly based on color distribution. No sophisticated image content analysis is included. However, not all color points are viewed equal in perception, and they should be clustered into different groups according to their visual importance so that different mapping strategies can be applied respectively to achieve a better color image reproduction. The proposed work introduces the perceptual image quality concept into gamut mapping of Color Management System by a multi-domain image gamut analysis. The ambiguity of rendering intent selection can be alleviated by content-aware image gamut analysis whose intention is to realize an Image Gamut Boundary Reduction (IGBR). It enables CMS a more reasonable selection of rendering intents and more coherent reproduction.
\end{abstract}

Index Terms-Color Management System, gamut mapping, ICC standard

\section{PROBLEM STATEMENT}

Color Management System (CMS) aims to realize coherent reproduction through a cross-media chain. It is a jointdomain issue which involves several areas especially color science, vision science and image processing. Many discussions and solutions can be found in literature [1] [2]. Most of the state-of-the-art CMS solutions are based on the International Color Consortium (ICC) [3] [4] standard Version 4 which was released at 2004 and proved as an International Standard ISO 15076-1 at 2005. CMS has become universally accepted and applied in both color and imaging industry.

Albeit with the development in color science and image processing, their incorporation is not yet sufficient in CMS domain due to the fact that most color laboratories focus more on physics and chemistry aspects by collecting empirical data from simple and isolated color stimuli (such as ink, paper and illuminations) [5] [6] rather than regional and content-based image level analysis; whereas, image processing and analysis approaches usually apply mathematical models for theoretical analysis rather than empirical experiences of color perceptions. Although some Human Vision System (HVS) properties have been roughly quantified, yet there are still large gaps to be bridged between color science and image processing in CMS issue.

In ICC V4 some recommendations have been made for cross-media reproduction such as color appearance model, chromatic adaptation, gamut mapping and rendering intents. Gamut mapping is regarded as the essential module for cross-media reproduction [7], and ICC V4 defines four rendering intents with two of them most applied to pictorial images which are Perceptual Rendering Intent (PRI) and Media-Relative Colorimetric Rendering Intent (MRCRI). Some gamut mapping algorithms can be used to realize these rendering intents such as SGCK for PRI and HPMINDE for MRCRI. However, these algorithms are mainly based on color distribution, which is pixel-level and unaware of image contents. There is also a frequent ambiguity of selecting either PRI or MRCRI during gamut mapping since the former preserves better contrast information which is considered to be essential to perception whereas the latter tends to keep more color fidelity during reproduction which is also decisive for color constancy.

More than 90 algorithms have been discussed in [7] most of which apply either gamut compression or clipping albeit with different varieties of HVS property integration and spatial extension to conventional GMAs. Besides these two categories, some pioneering work was carried out by S.Nakauchi et al. in 1996 to model gamut mapping as an optimization problem [8]. This approach is revisited in 2007 by J.Giesen et al. in [9]. However, the success of these methods depends heavily on the accuracy of the difference metrics being minimized.

As a matter of fact, most state-of-the-art GMAs or SGMAs focus only on the gamut mismatch problem. Little concern has been put on image gamut itself. Almost all proposals apply the whole color set of input image as source data which may be inappropriate since not all color difference is visible and not all color points are viewed equally in perception. Therefore, the insistence of applying the whole color set restricts their performances in solving gamut mismatch problem.

The structure of this paper is organized into six sections including the current problem statement. The motivation is given in next section explaining the objective of this work. 
Section three describes the outline of the proposal followed by detailed discussion of implementation in section four. Some test results are shown in three different cases in section five. At last summary is made in section six, followed by references.

\section{MOTIVATIONS}

The concept developed in this work does not aim to bring out a new gamut mapping algorithm, but to enable the stateof-the-art GMAs or SGMAs with better performance by an image content analysis. With integration of the proposed IGBR as a pre-processing of gamut mapping, it is likely to reduce color distortions introduced by the existing solutions. Due to rapid developing progress of gamut mapping algorithms, standard ICC-based approaches will be applied in this work, but the concept introduced is transferable to other GMAs and SGMAs in applications.

The proposed work is inspired by perceptual approach of Image Quality Assessment (IQA) whose purpose is to account for only visually important information during image quality ranking. It carries out a multi-domain (spatial, color and perception) analysis whose objective is to reduce effective image gamut boundary before gamut mapping. This operation removes perceptually less important regions from the out-of-gamut (OOG) color distribution so that it contains only essential contents for gamut mapping. IGBR enables less gamut compression during color image reproduction and more reasonable rendering intent selection based on image contents' relative importance.

\section{METHODOLOGY}

For the OOG color points, a spatial domain analysis examines the geometrically dispersed color points which do not group into visually important clusters. It is well known that HVS spatial vision tends to make fusions of small dots into regions to form perception of objects, based on which the half-toning printing technology is realized. This means that these isolated color points could possibly be replaced by Inside-Gamut (IG) points without damaging perceptual image quality due to their geometrical disparity. However, the image gamut boundary indeed shrinks due to the reduction of OOG points, and by consequence less gamut compression is necessitated since there are fewer points to be mapped.

Similarly, image gamut reduction is also carried out in color domain. Some colors points are extremely saturated but very rare in presence. Nevertheless, they belong to the most influencing group in color distribution based on which the image gamut boundary is defined! However, these few extremely saturated points may be resulted from artifacts such as salt-and-pepper noise or camera acquisition errors, or even if they indeed belong to image content but too few to be remarkable. Replacing them by IG points actually does not damage global perception of image appearance, whereas the color reduction does reduce considerably the image gamut boundary. The removal of these extreme points usually has decisive impact to compression-based gamut mapping algorithms.

Last but not least, different regions have different visual importance to human beings. HVS is usually keener to dramatic lightness and chroma variations compared to gradually changed area. Therefore, among the OOG color areas, the salient parts need more cautious preservation due to their sensitivities to distortion, whereas the non-salient parts could be possibly replaced by IG colors without drawing too much attention. The removal of non-salient regions also enables an image gamut reduction which leads to less perceptual distortion during gamut compression.

After multi-domain IGBR, most visually less important OOG color points have been replaced by their closest IG colors without damaging severely the perceptual appearance. These reduced color points include geometrically isolated colors, extremely saturated (but few in presences) colors, and the color points from non-salient areas. Through IGBR, a direct gamut mapping without clipping or compressing is possible if the reduced image gamut boundary fortunately falls into output device gamut, or an ICC Media-Relative Rendering Intent can be applied for better preserving hue constancy if the remaining OOG points are not in considerable number, or an ICC Perceptual Rendering Intent can be used to keep contrast information if the OOG points still remains remarkably abundant.

\section{IMPLEMENTATION}

\subsection{Extracting the OOG points}

Although the "Gamut" tag in ICC header file can be used to extract OOG regions, it is not always supported well in some profiles such as RSWOP.icm for printers according to our test. In this work, the OOG is calculated using the color difference between the original image and its soft proof version. Media-Relative Colorimetric Rendering Intent (MRCRI) is adopted for generating soft proof since it preserves better the hue constancy for the IG colors. The aforementioned Multi-domain IGBR is applied to this calculated OOG data.

For the most generality, the input ICC profile uses sRGB which is a Microsoft standard aiming to describe the minimum intersection of the gamuts of most commonly used displays, and the output ICC profile applies RSWOP which is default printer profile in MS Windows system. Figure 1 shows an example of the original image and its soft-proof using MRCRI. As can be seen, the color appearance is changed due to gamut limit of the printer. Figure 2 shows gamut visualization in color space CIELab for clearer comparison. The empty circle points are the gamut boundary of printer. We can find some extremely saturated points of image gamut (shown as filled circle points) are beyond the 
gamut boundary of printer which means that they cannot be reproduced for being out of the reproducibility of printer. Figure 3 shows the extracted OOG regions for this image.

However, HVS has certain tolerance for color variation which means not all color difference is visible. The color tolerance can be calculated using CIE DE2000 formulas [10]. Generally, if the color difference in Delta E2000 is larger than 3, most normal observers are capable to discern it. We apply this threshold to OOG regions to extract only visible color points.
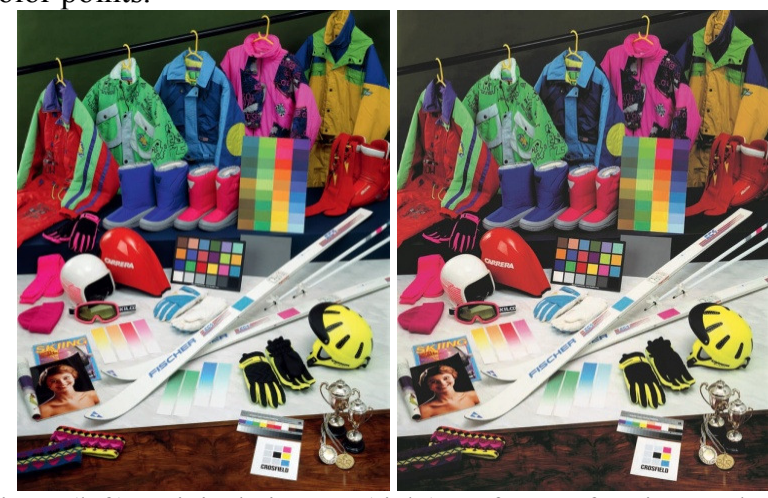

Fig.1 (left) original image (right) soft proof using Relative Rendering Intent

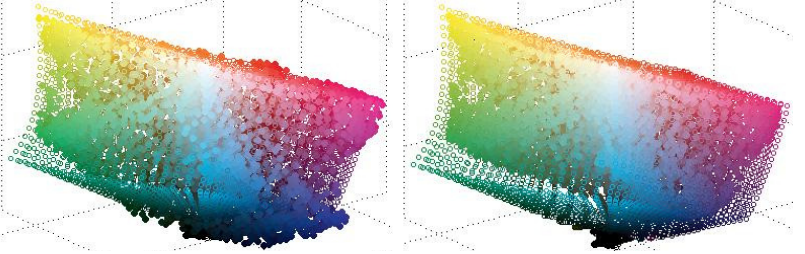

Fig.2: Color distribution of above images
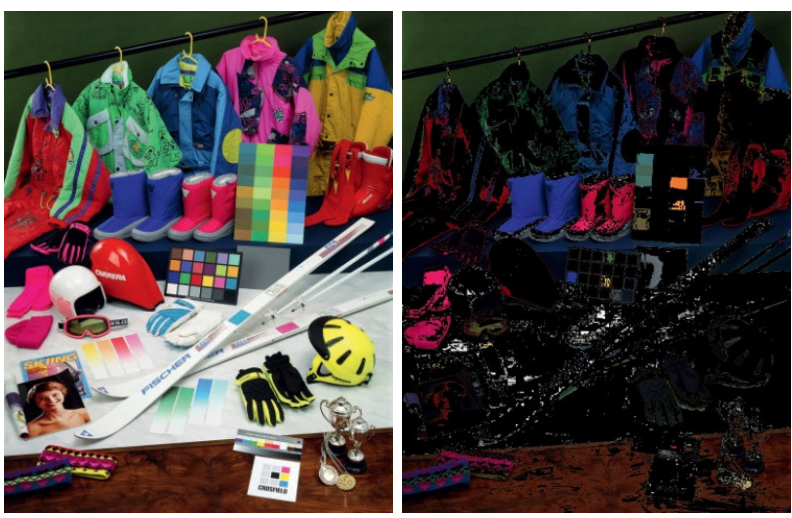

Fig 3 (left) original image (right) visible OOG regions

\subsection{Spatial reduction of image gamut boundary}

Spatial domain analysis is applied to OOG regions to examine the geometrically dispersed color points which do not group into visually important clusters. A morphological image opening operation is used to eat up the isolated color points. The structural element used is in shape of diamond with distance from origin to points of diamond equals to 4 . Figure 4 shows the obtained OOG regions after spatial reduction. Figure 5 shows the resulted image after OOG spatial reduction. We can hardly find any visible differences between them whereas the resulted image does have its gamut boundary reduced as indicated by the color distribution comparison. This leads to less impact to image content during gamut compression since there are fewer points to be mapped.
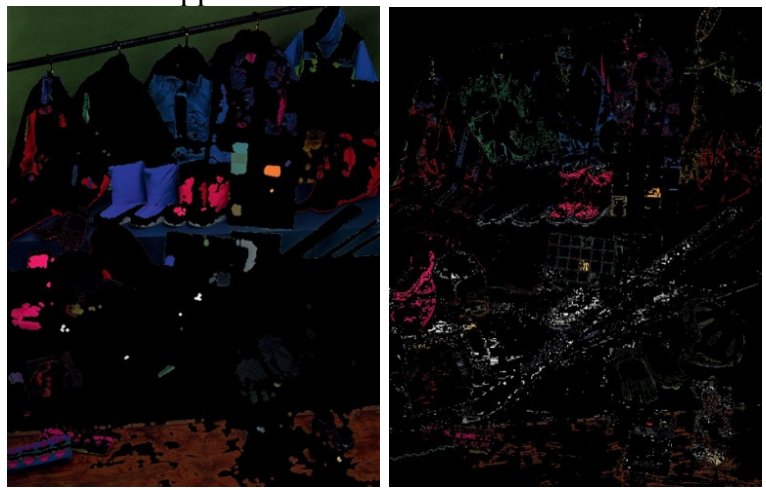

Fig.4 (left) OOG regions after image opening (right) the difference before and after image opening (which are the color points to be clipped to IG.)
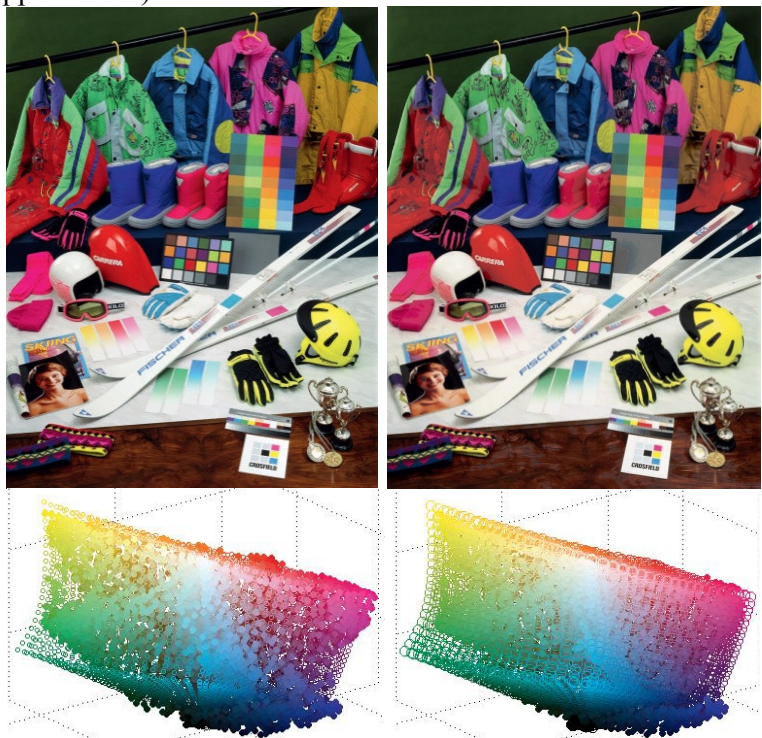

Fig.5 (1st column) original image and its gamut (2nd column) image after spatial reduction and its gamut

\subsection{Color reduction of image gamut boundary}

The resulted image after spatial reduction is converted to CIELCh color space whose advantage is direct access to lightness and chroma components. 5\% most saturated OOG points will be clipped into IG area. These color points are selected using histogram counting in chroma component. Figure 6 shows over saturated points after spatial reduction.

Despite of their high values in chrominance, these 5\% over-saturated points are less impactful in global perception due to their few presences. Therefore, replacing them by the closest IG color points can tremendously reduce image gamut boundary. Figure 7 shows the resulted image and its 
gamut after spatial and color reduction. Few visible differences have been caused due to color reduction whereas the image gamut boundary does be reduced.
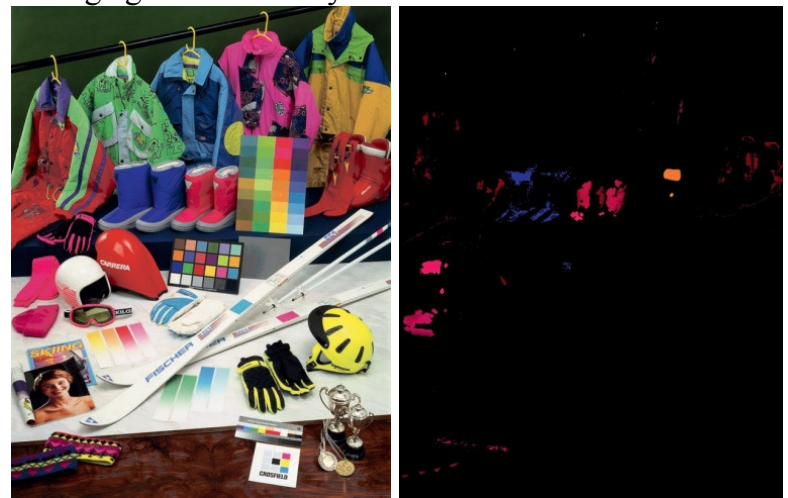

Fig.6 (left) Image after spatial reduction (right) the 5\% of most saturated color points
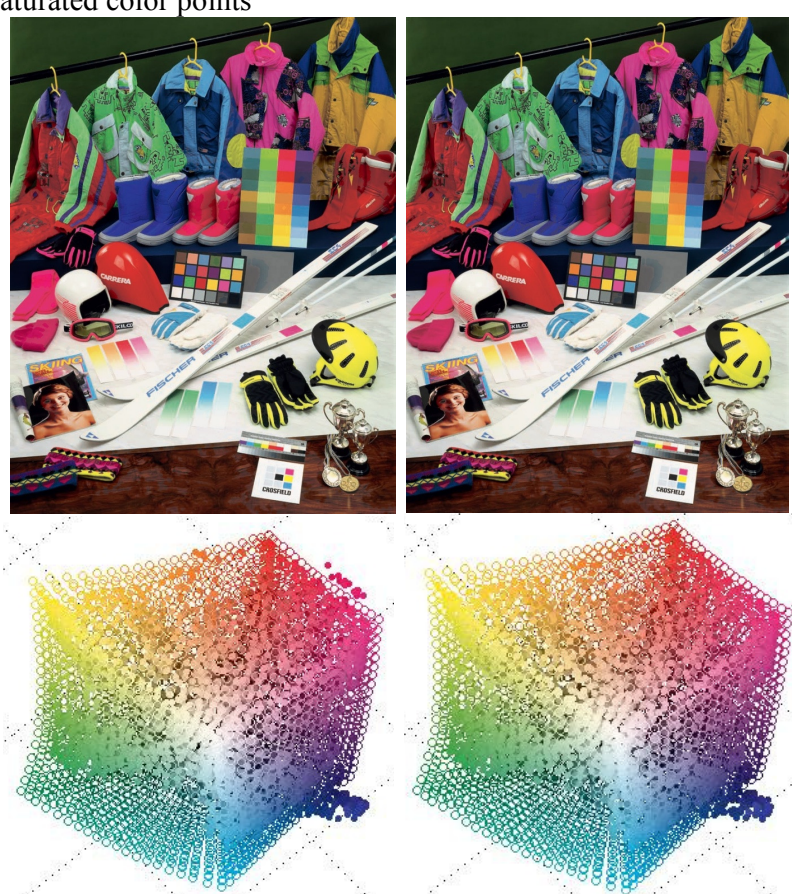

Fig.7 (1st column) image after spatial reduction and its gamut (2nd column) image after spatial and color reduction and its gamut. The image gamut is viewed from the top.

4.4 Non-salient region reduction of image gamut boundary This work applies algorithm proposed by R.Achanta et al. [11] for saliency detection thanks to its intuitive design and continuity of contour in saliency detection. A typical saliency map example is shown in figure 8 . With application of this algorithm, salient regions after spatial reduction and color reduction can be extracted as shown in figure 9 . Note that the mean value of saliency map is used as threshold to separate salient and non-salient regions. Figure 10 shows the image and its gamut before and after non-salient regions reduction. Although some area has visible difference after mapping OOG non-saliency regions to IG due to physical limit of device, ye since the prominent component is not touched, non-salient OOG color removal does not damage severely the perceptual quality while enabling an image gamut boundary reduction which leads to less perceptual distortion during gamut mapping.
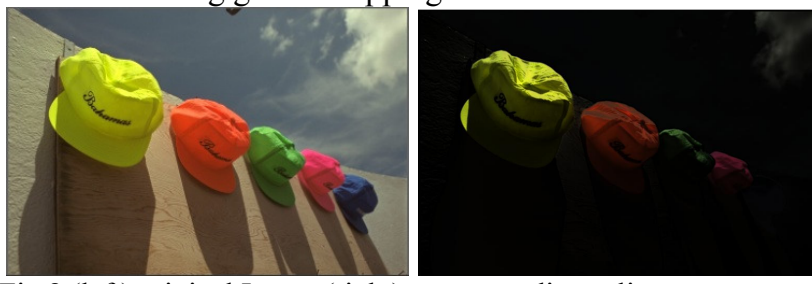

Fig.8 (left) original Image (right) corresponding saliency map
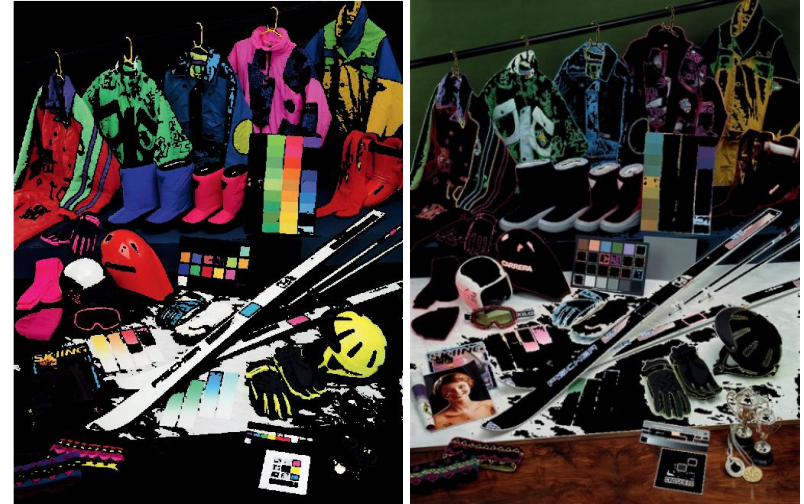

Fig.9 (left) silent regions after spatial and color reduction (right) non salient counterpart.
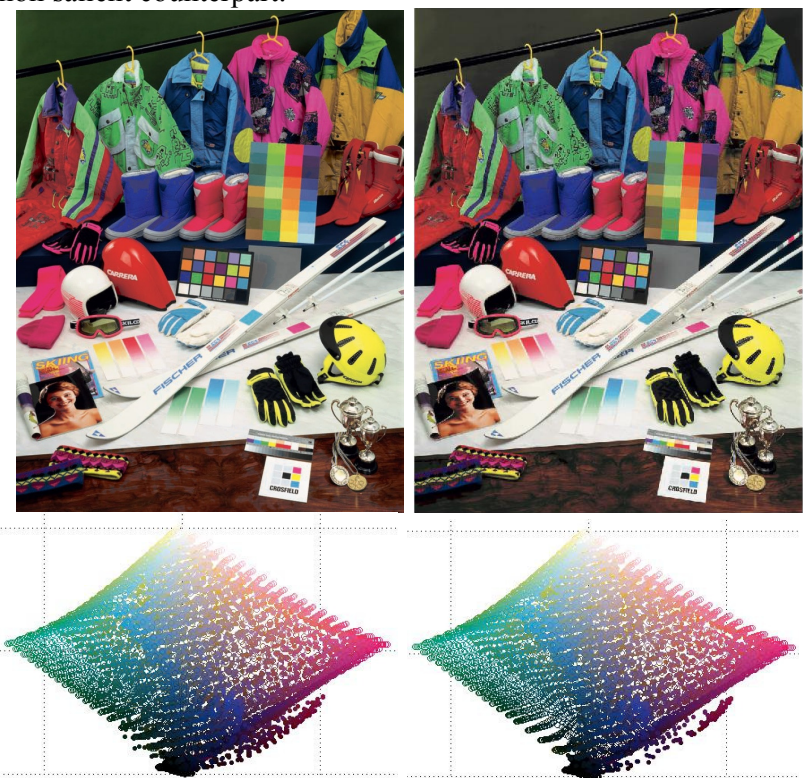

Fig.10 (1st column) image after spatial and color reduction and its gamut (2nd column) result after one more step of mapping OOG non-salient regions to IG. The image gamut is viewed from side.

\subsection{Gamut clipping for the remaining $O O G$ points}

After multi-domain IGBR, most visually less important color points have been replaced by their closest IG color points without damaging too much the perceptual appearance. These reduced color points include geometrically isolated 
colors, extremely saturated (but few in presences) colors, and the color points from non-salient area. Another gamut clipping servers as post-treatment to ensure that all the remaining OOG points are clipped into IG after IGBR.

\section{EXPERIMENTS AND RESULTS}

\subsection{Direct gamut mapping}

If image gamut after IGBR fortunately falls into output device gamut, a direct gamut mapping without clipping or compressing is possible since all colors are reproducible now. This maximizes the perceptual coherence. The image can be sent to printer directly after a Chromatic Adaptation without gamut mapping. Figure 11 shows such an example with the original image and its IGBR version. A comparison of this result with general ICC perceptual rendering result is shown in figure 12. On observing the results, we can find that the direct reproduction after IGBR tends to preserves more color constancy for image contents.
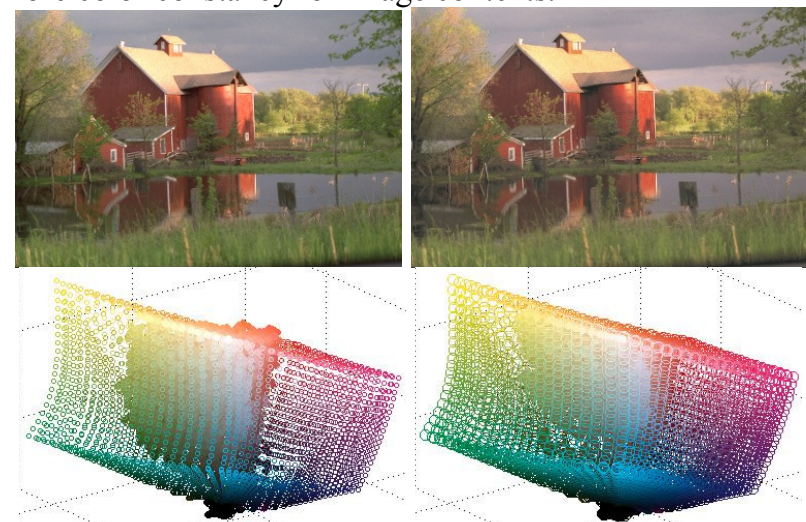

Fig. 11 ( $1^{\text {st }}$ column $)$ original image and its gamut $\left(2^{\text {nd }}\right.$ column $)$ result by multi-domain IGBR and its gamut
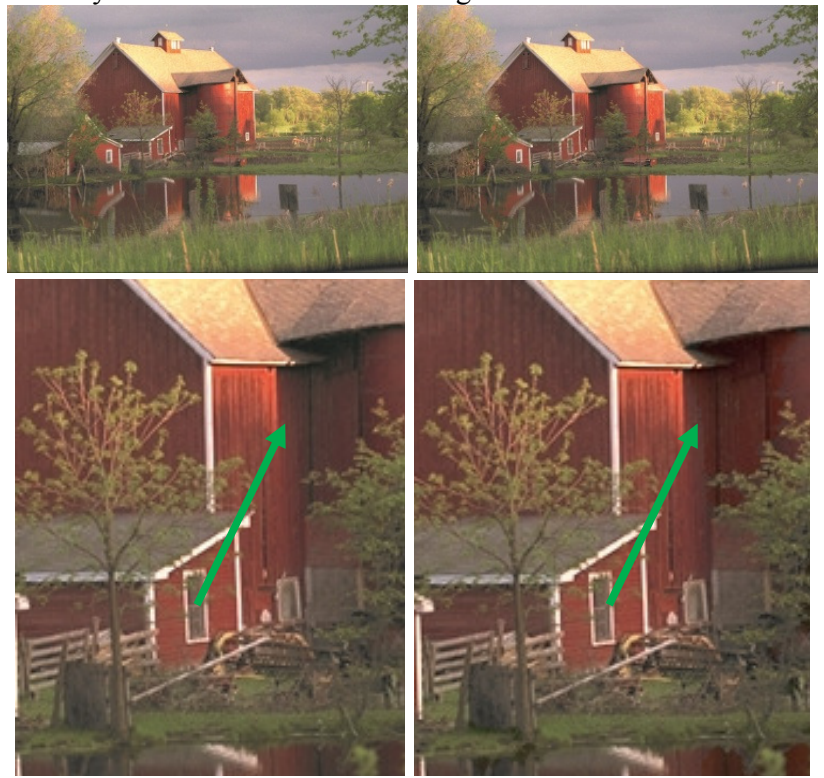

Fig.12 (1st column) result by ICC Perceptual Rendering Intent and its zoomed region (2nd column) direct reproduction after IGBR

\subsection{Relative Rendering Intent for gamut clipping}

If the remaining OOG points are not in great number compared to image size, a Media-Relative Colorimetric Rendering Intent (MRCRI) is appropriate to better preserve hue constancy. In this work, $10 \%$ is used as threshold which means that if the remaining OOG points after IGBR are no more than $10 \%$ of image size, then MRCRI will be used under which case a Perceptual Rendering Intent (PRI) is usually recommended by ICC. However, it is not wise to compress everything outside gamut before analyzing their importance. Figure 13 shows an example of this case. Some close-up comparison is shown in figure 14.
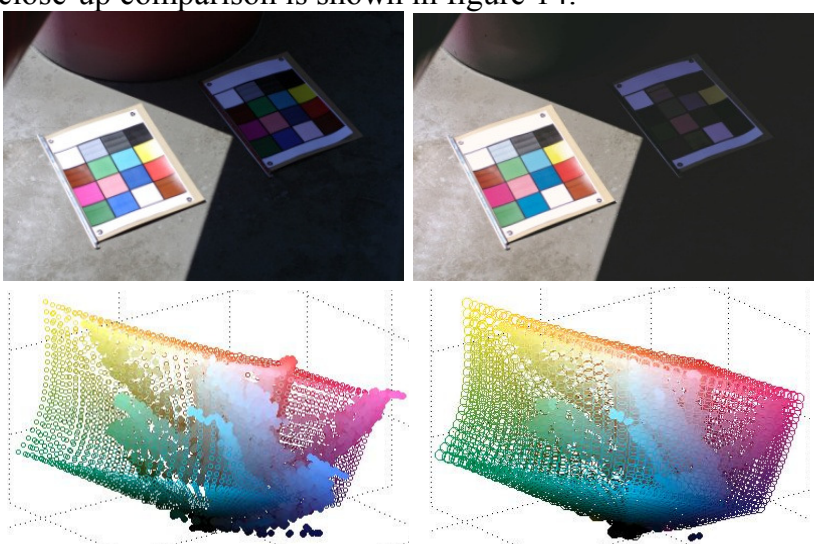

Fig.13 (1st column) original image and its gamut (2nd column) image result from multi-domain IGBR and its gamut visualization

\subsection{Perceptual Rendering Intent for gamut compression}

If, unfortunately, the remaining OOG points after IGBR are still in large amount (more than $10 \%$ of image size), Perceptual Rendering Intent (PRI) should be appropriate since the remaining regions contain essential image content. Figure 15 shows such a case where PRI can better preserve contrast information after IGBR operation. Some close-up views are included in the figure. The image result of gamut mapping by Perceptual Rendering Intent after IGBR is beneficiated from both gamut clipping for the visually unimportant regions and gamut compressing for the visually important ones as shown by arrows on the figure.

\section{SUMMARY}

The proposed work introduces the perceptual image quality concept into gamut mapping of Color Management System by a multi-domain image gamut analysis. By calculating the effective gamut boundary, an Image Gamut Boundary Reduction (IGBR) is realized thanks to which the ambiguity of rendering intent selection can be alleviated and less impact can be caused during gamut mapping since there are fewer color points to be mapped. By this means, gamut mapping process accounts for not only color attributes (lowlevel information), but also their spatial distribution (midlevel features) and visual saliency (high-level property). It 

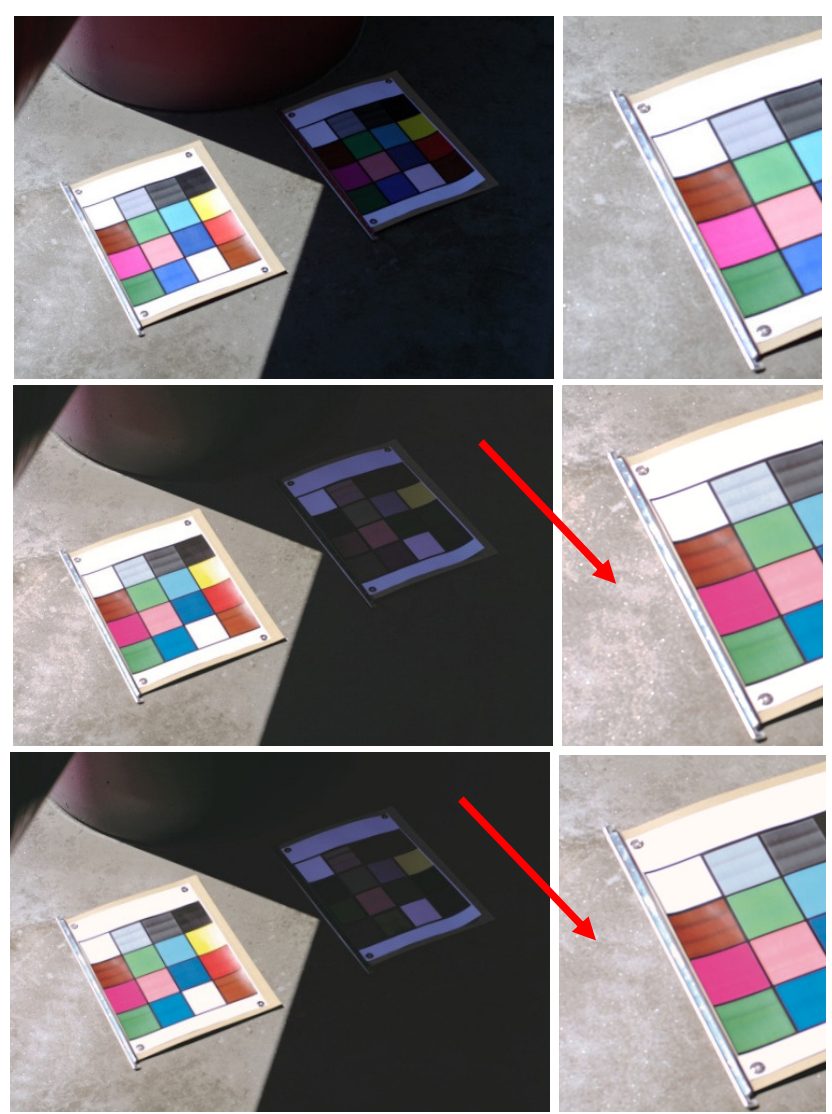

Fig.14 $\left(1^{\text {st }}\right.$ row $)$ original image and its zoomed region $\left(2^{\text {nd }}\right.$ row $)$ result by ICC Perceptual Rendering Intent (3rd row) result by Media-Relative Colorimetric Rendering Intent after IGBR
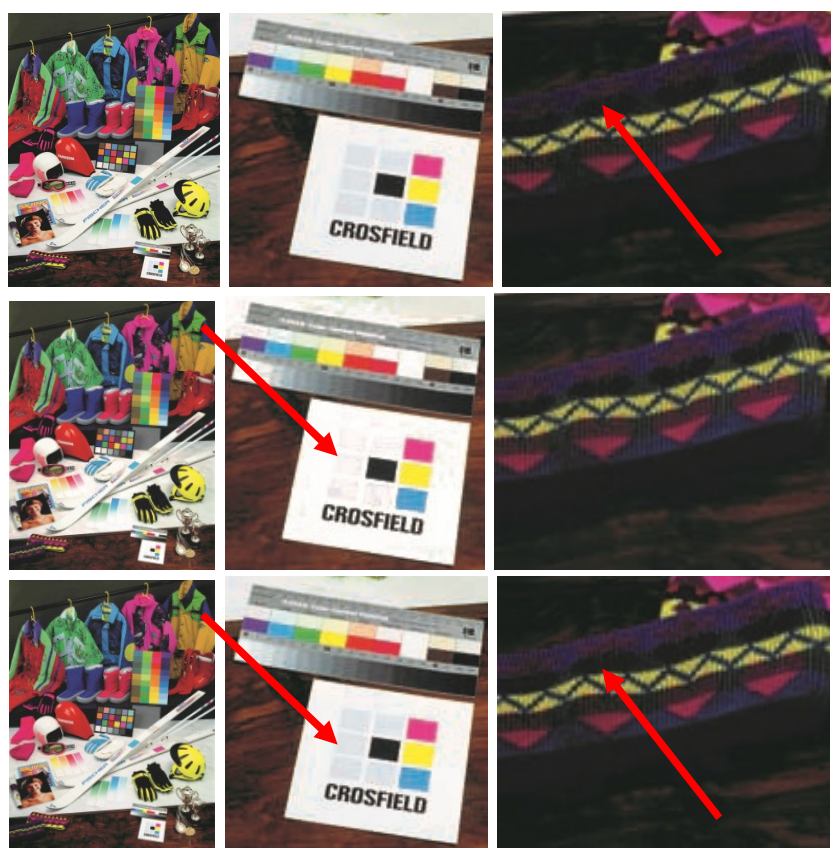

Fig 15 (1st row) result by Media-Relative Colorimetric Rendering Intent (2nd row) result by Perceptual Rendering Intent (3rd row) result by Perceptual Rendering Intent after IGBR operation enables CMS with a more reasonable selection of rendering intents and a more coherent reproduction.

Limited by the immaturity of the proposal, the multidomain IGBR brings only moderate improvement to color constancy at current stage, yet it initiates another issue on content-based gamut mapping by image gamut reduction where more fruitful consecutive works can be anticipated.

\section{REFERENCES}

[1] B.Fraser, C.Murphy, and F.Bunting, "Real World Color Management (2nd edition)", Peachpit Press, 2004

[2] J.P Homann, "Digital Color Management: Principales and Strategies for the Standardized Print Production", Springer, 2008

[3] http://www.color.org, and the ICC standard version 4 homepage: http://www.color.org/v4spec.xalter

[4] P.Green, and M.Kriss, "Color Management: Understanding and Using ICC Profiles", Wiley, 2010

[5] M. D. Fairchild, "Color Appearance Models (2nd edition)", Wiley, 2005

[6] R.W.G. Hunt, "The Reproduction of Color, 6th edition", Wiley, 2004

[7] J.Morovic, "Color Gamut Mapping", Wiley, 2008

[8] S.Nakauchi, M.Imamura and S.Usui, "Color gamut mapping by optimizing perceptual image quality", $4^{\text {th }}$ Color Imaging Conference: Color Science, System, and Applications, 1996

[9] J.Giesen, E.Schuberth, K,Simon, P.Zolliker and O.Zweifel, "Image-dependent gamut mapping as optimization problem", IEEE Transactions on Image Processing, Oct. 2007

[10] G.M.Johnson, M.D.Fairchild, “A Top Down Description of SCIELAB and CIEDE2000,” Color Res. Appl. 27, 2002

[11] R. Achanta, S. Hemami, F. Estrada and S. Süsstrunk, "Frequency-tuned Salient Region Detection", IEEE International Conference on Computer Vision and Pattern Recognition (CVPR), 2009 